\title{
Drug Development: From Concept to Marketing!
}

\author{
Nihad A.M. Tamimi Peter Ellis \\ Pfizer Inc., Sandwich, UK
}

\section{Key Words}

Drug discovery $\cdot$ Clinical trials $\cdot$ Drug approval $\cdot$ Drug safety

\begin{abstract}
Drug development is an expensive, long and high-risk business taking 10-15 years and is associated with a high attrition rate. It is driven by medical need, disease prevalence and the likelihood of success. Drug candidate selection is an iterative process between chemistry and biology, refining the molecular properties until a compound suitable for advancing to man is found. Typically, about one in a thousand synthesised compounds is ever selected for progression to the clinic. Prior to administration to humans, the pharmacology and biochemistry of the drug is established using an extensive range of in vitro and in vivo test procedures. It is also a regulatory requirement that the drug is administered to animals to assess its safety. Later-stage animal testing is also required to assess carcinogenicity and effects on the reproductive system. Clinical phases of drug development include phase I in healthy volunteers to assess primarily pharmacokinetics, safety and toleration, phase II in a cohort of patients with the target disease to establish efficacy and dose-response relationship and large-scale phase III studies to confirm safety and efficacy. Experience tells us that approximately only 1 in 10 drugs that start the clinical phase will make it to the market. Each drug must demonstrate safe-
\end{abstract}

\section{KARGER}

Fax +4161306 1234 E-Mail karger@karger.ch www.karger.com
(C) 2009 S. Karger AG, Basel

$1660-2110 / 09 / 1133-0125 \$ 26.00 / 0$

Accessible online at:

www.karger.com/nec ty and efficacy in the intended patient population and its benefits must outweigh its risks before it will be approved by the regulatory agencies. Strict regulatory standards govern the conduct of pre-clinical and clinical trials as well as the manufacturing of pharmaceutical products. The assessment of the new medicinal product's safety continues beyond the initial drug approval through post-marketing monitoring of adverse events.

Copyright $\odot 2009$ S. Karger AG, Basel

\section{Introduction}

Getting drugs to the market is an expensive and highrisk business which takes on average $10-15$ years to complete. The Tufts Center for the Study of Drug Development announced in November 2001 that the average cost to develop a new prescription drug was USD 802 million [1]. When the costs of failed prospective drugs are factored in, the actual cost for discovering, developing and launching a single new drug would have exceeded 1.5 billion. This compares with USD 4 million in 1962 and USD 231 million in 1987 [2, 3]. The problem is compounded by the high attrition rate, as it is estimated that approximately only 1 in 10 drugs that enter clinical trials will make it to the market. In a recent study, it was shown that the average success rate for drugs to be approved for all therapeu- 
Fig. 1. Phases of drug development.

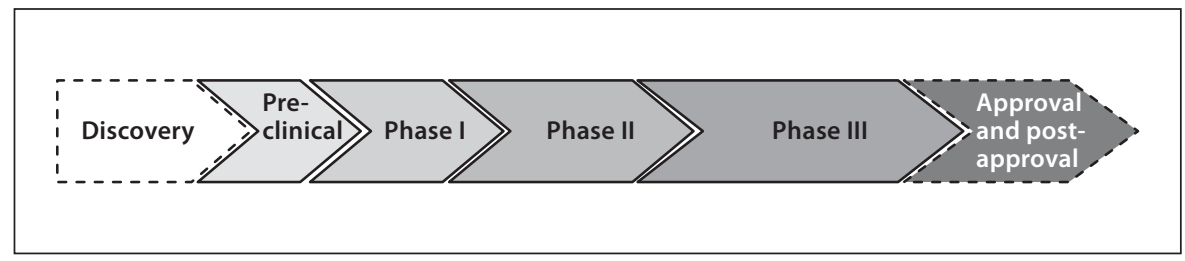

Fig. 2. Drivers for discovering new drugs with examples.

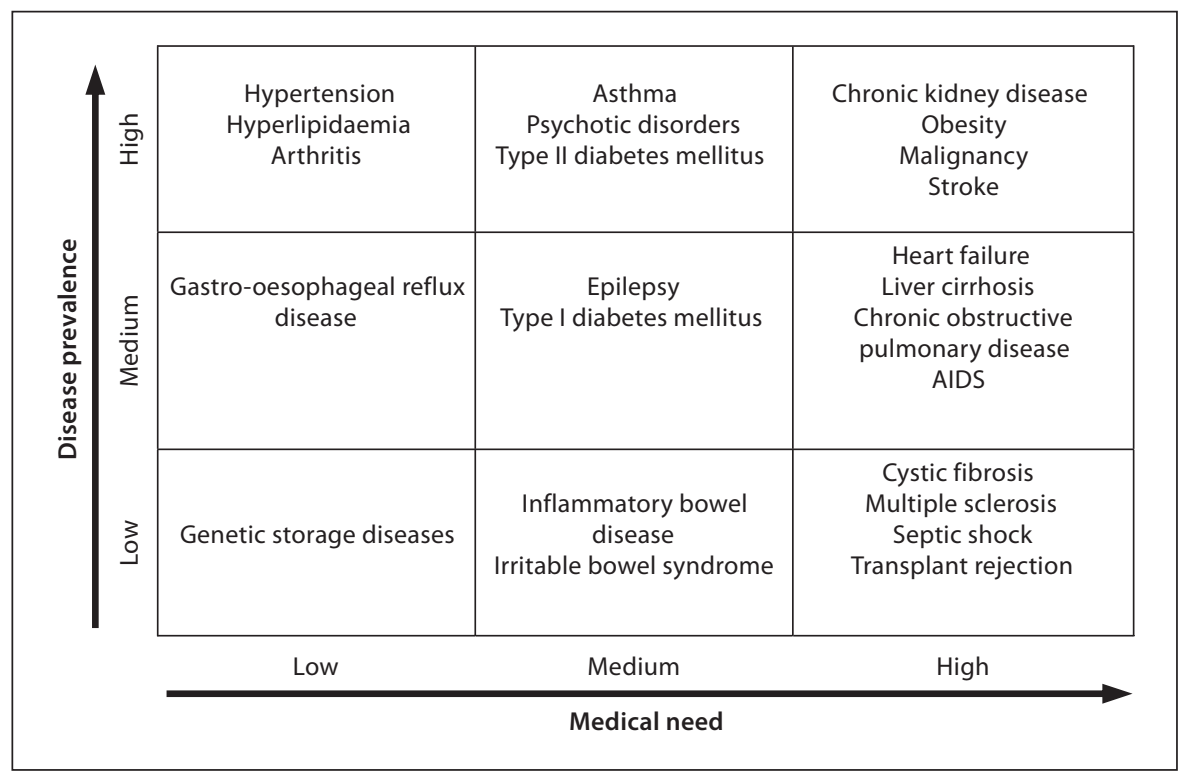

tic areas is approximately $11 \%$. The success rate varies between therapeutic areas ranging from $20 \%$ for cardiovascular drugs to only $5-8 \%$ for oncology and central nervous system disorder drugs [4]. Improvements in predicting the potential success or failure of a product in clinical trials is essential to aid in reducing the spiralling development costs. Unfortunately, increasing costs combined with the high attrition rate are forcing pharmaceutical companies to reduce investment in research and development, focussing on a more limited product portfolio.

Drug development is a significant challenge. Every product must not only be safe and efficacious, but its efficacy has also to be proven across racial and ethnic groups as well as across different age groups. Every drug has to pass a global regulatory review in what is currently the most regulated industry in the world. Once this is done, approved products must appeal to global markets across different cultures, healthcare systems and distribution systems.

It is interesting to note that in a recent survey, the public perception was that the pharmaceutical industry dis- covers only $27 \%$ of new drugs whilst the reality is that more than $90 \%$ of all new drugs are discovered by the industry [5].

This minireview will address the process of drug development from discovery through the stages of development up to approval and marketing (fig. 1).

\section{Discovery}

Selecting therapeutic areas or indications to invest in is driven by 'medical need' and the prevalence of the disease (fig. 2). Additional factors also include technical feasibility, research and development costs and commercial considerations such as competition in the market place and potential market share. Even if these criteria are met, there is only a limited research and development budget and each new project must be prioritized against the company research and development portfolio, with only high priority projects within the budget being selected for progression. For many companies, this is typically an an- 
nual review process for products at all stages of development. This may lead to stopping a programme even at an advanced stage of development.

Early chemical starting points have been identified from naturally occurring substances in plants, humans or animals but lead compounds are more often sourced from targeted chemical synthesis directed to bind to the known structures of receptors and enzymes or from random or receptor-targeted high-throughput screening [6]. This has become more popular in the last few years as it is helpful in accelerating drug discovery. Initial problems encountered in the last decade have eased with improving technology. With the advent of modern computer technology, robotics and multi-well assay plates (384 growing to 1,536 wells per plate), high-throughput screening can test vast 'libraries' of chemical compounds in multiple screens (which can deliver up to 120,000 assays every $24 \mathrm{~h}$ ). Another method of lead identification is 'virtual screening' (also named in silico screening) which is defined as the 'selection of compounds by evaluating their desirability in a computational model' [7]. Compounds testing positive in screening have their potency and selectivity confirmed by in vitro biochemical or cellular assays. This is typically followed by functional biochemical and pharmacological testing in vitro, followed by pharmacodynamic and pharmacokinetic testing in vitro and in vivo [8]. The next step is to complete pilot toxicology testing to inform us of the likely safety profile. Once all preclinical testing has satisfied the minimum selection criteria, the compound transitions from a 'lead' to a 'candidate' and is nominated for progression to the clinic.

At this stage, drug production is scaled up to meet the increased compound demand, work commences on developing a suitable formulation for clinical use (often a tablet is the preferred dosage form) and the candidate is progressed through the required toxicology testing (including genotoxicity, safety pharmacology in all biological systems, single and multiple dose toxicity and toxicokinetic studies) to enable the first in human and subsequent clinical studies. Reproductive toxicology in male and female animals (required prior to testing in women of child-bearing potential) and long-term carcinogenicity testing are also prerequisites for filing a drug approval request [9].

In parallel with lead development/candidate nomination, a key decision on when to patent the compound or chemical series is taken. Early patenting mitigates against competitors beating a company to a claim, but delaying the patent application allows for introduction of additional data to strengthen the patent and extends the pat- ent expiry date. The patent life is typically 25 years but as it takes 10-15 years to develop a drug, there could only be 10 years remaining to sell the product and recoup the high development costs.

\section{Phases of Clinical Drug Development}

Phase I. Phase I starts with the first administration of the new medicinal product to humans. Usually this phase involves healthy volunteers with the exception of cytotoxic drugs (e.g. oncology drugs) which get tested in patients without the requirement to test in healthy volunteers first. The purpose of this stage is to evaluate the safety, tolerability, pharmacodynamic (effect of the drug on the body e.g. effect on heart rate, blood pressure, electrocardiogram (ECG), etc.) and pharmacokinetic (effect of the body on the drug i.e. absorption, distribution, metabolism and excretion) effects of the tested drug. Phase I studies are usually conducted in dedicated phase I units which are research units attached to a general or teaching hospital and manned by research physicians who are familiar with conducting such studies. Full resuscitation facilities are available at these units. Phase I studies require approval from an ethics committee and the relevant regulatory agency. In the United States, an Investigational New Drug (IND) application, which summarises the established preclinical and manufacturing information along with investigator guidance, must be in place prior to starting clinical trials. A pre-IND consultation programme is offered by the US Food and Drug Administration (FDA) to provide guidance on the data necessary for the IND submission. Subjects are usually compensated for participating in these studies. Development of the drug could be stopped if it is found that the half-life of the drug is too short or too long or if it has poor bioavailability. Similarly, if the drug is not well tolerated at effective concentrations it is dropped from development. Phase I studies usually start with single sub-pharmacological doses which are escalated gradually and followed by multiple doses. Stopping rules to dose escalation include severe adverse events, clinically significant ECG abnormalities and clinically significant laboratory abnormalities.

Other phase I studies to support drug development are conducted throughout phase II and II of development. These include drug-drug interaction studies, effect of food on absorption, age and genetic influences. A typical phase I study can cost up to USD 500,000, with speciality studies (such as detailed QTc ECG assessments) costing up to USD 1.5 million. 


\section{Role of Translational Medicine/Biomarkers}

The American Physiological Society has defined translational research as 'the transfer of knowledge gained from basic research to new and improved methods of preventing, diagnosing, or treating disease, as well as the transfer of clinical insights into hypotheses that can be tested and validated in the basic research laboratory' [10]. Biomarkers are quantitative measures of biological effects that provide informative links between mechanisms of action and clinical effectiveness [11]. Effectively applying translational research measures to a development programme in phase I and phase II results in earlier identification of efficacy (or just as important, lack of efficacy) resulting in increased overall productivity and potentially a quicker route to drug approval. There are 3 fundamental classifications of biomarkers: (1) markers of disease e.g. proteinuria as a biomarker of chronic kidney disease (CKD); (2) markers of pharmacological activity of a drug e.g. inhibition of angiotensin-converting enzyme increases plasma levels of angiotensin-1 and decreases plasma levels of angiotensin-2; (3) surrogate biomarkers of efficacy e.g. using a measure of penile rigidity measured by plethysmography (Rigiscan) as a surrogate for sexual intercourse. An example of a biomarker with diagnostic rather than efficacy potential is neutrophil gelatinase-associated lipocalin or NGAL, which serves as biomarker of acute renal injury as increased levels are detected in urine and blood within hours of kidney injury.

Taking the example of proteinuria in CKD, interventions that reduce proteinuria can be potentially beneficial in the treatment of CKD. Therefore measuring changes in the biomarker in both preclinical models (e.g. sub-total nephrectomy model in the rat) and the clinic can be indicative of activity of a potentially new drug for treating that indication (i.e. slowing progression of non-diabetic CKD). The challenge is to use or develop a biomarker in which we have confidence that it will reflect changes in the important registrable endpoints that we will assess in phase III trials and which are essential to gain regulatory approval.

Phase II. Once the drug's safety, pharmacokinetics and dose selection has been established in healthy volunteers, the next step is to investigate the efficacy and safety of the drug in the target population. For example, if a drug is being developed for the treatment of hypertension, phase II trials will involve investigating the drug in a hypertensive patient population. Phase II is usually divided into phase IIa and phase IIb. Phase IIa is when the drug (usually limited to a single high/maximal tolerated dose level) is tested in a small cohort (12-100) of patients; this is called the 'proof of concept'. Phase IIb follows on from the proof of concept in which several dose levels are tested in the target population (dose-ranging studies) to define the minimally effective or non-effective dose and to decide the optimal dose, based on clinical efficacy and safety, to take to the next stage. Occasionally phases IIa and IIb are combined in one large study. A complete phase II programme could involve several hundred patients and can cost several million dollars.

With ever increasing development costs and expiry of valuable patents on major products, the pharmaceutical industry is compelled to develop more efficient and costeffective ways of doing drug development. These include the use of biomarkers, as discussed, but also application of enhanced quantitative drug design 'EQDD' to understand exposure-response relationships and optimise dose selection, thus facilitating regulatory review and maximising the commercial value of the drug.

However, positive phase II data is no guarantee of progression to phase III. At this key stage of development, costs will increase significantly and detailed analyses of the drug candidate and the market (patient, payer and physician perspectives) are conducted. This will include drug efficacy relative to the competitors, safety profile, probability of technical and regulatory success, remaining patent life of the drug, cost of goods to produce the drug, potential market share and pricing and reimbursement. Once again, the drug will be prioritised against all other candidates in the portfolio and only if the outlook is favourable and the priority is within the research and development budget will it go forward.

A successful phase II is followed by an 'end of phase II' meeting with regulatory agencies such as the FDA to discuss the results from phase II and discuss and agree the clinical and statistical analysis plans for phase III. This negotiation, which also includes the target labelling, is critical to ensure alignment between the regulatory agency and sponsor.

Phase III. This is the final stage of drug development prior to registration and will confirm the clinical doses, frequency and timing of administration for approval. Before embarking on a costly phase III programme, the sponsor should have a high level of confidence in the drug's safety and efficacy in the target patient population and the dose range to be tested. Phase III trials (usually a minimum of 2) can involve up to several thousands of patients, depending on the indication, so that an adequate database (with $90 \%$ power to detect statistically significant differences) is created to assess the efficacy and safety profile, in addition to enabling accurate drug label- 
ling. Phase III trials are primarily designed and powered to test the hypothesis of efficacy but at the same time, adverse events are collected to assess benefit-risk potential of the drug. Use of novel endpoints in phase III is a highrisk strategy, but can prove valuable in demonstrating benefits relative to competitors or established therapies; however, these endpoints do require validation and should be included in phase II and discussed with the regulatory authorities prior to the start of phase III.

Clinical studies that use mortality and morbidity endpoints are often very large and can take several years to complete. Oncology is an exception, with phase III studies often limited to a few hundred patients. In diseases in which there is an established 'gold standard' treatment, European regulatory authorities will require phase III studies to include a comparator arm to demonstrate noninferiority or superiority compared to the standard therapy. Efficacy can be demonstrated either by demonstrating superiority to placebo in placebo-controlled trials or by showing superiority to an active-control treatment. Sometimes the new drug entity is compared to a reference treatment without the objective of showing superiority. This can be either an equivalence trial, which shows that the response to treatments differs by an amount which is clinically not significant (specify upper and lower equivalence margins), or a non-inferiority trial which has the objective of showing that the new drug is not clinically inferior to the comparator (only lower equivalence margin is specified). The choice of specified margins should be clinically justified.

Depending on the nature of the study and the endpoints used for the indication, a 'Data Safety Monitoring Board' (DSMB) may be required throughout the conduct of the trial. This is especially so in studies that incorporate mortality and morbidity as primary or secondary endpoints. DSMB members must include a clinician with expertise in the disease area under investigation as well as a biostatistician as a minimum. Each DSMB must have a charter and written operating procedures detailing members' responsibilities and the plan of communication. DSMB members must disclose potential conflict of interest to the sponsor.

For the sponsor, phase III trials involve a large crossfunctional team which involves, amongst others, clinicians, project management, data management, drug safety monitoring, document management, regulatory support and clinical quality assurance. A key consideration for phase III is selection of study centres to ensure appropriate patient recruitment and timely completion of the study. Estimations of patient drop-out rates are made, but if the rate is too high, additional study centres will be recruited. This ensures adequate patient numbers for approval, but is costly in incurring delays to the programme. The overall success rate of phase III is around $70 \%$ and depending on the size can cost up to USD 100 million. A successful phase III is usually recognised by the financial markets with an impact on the sponsor's share price.

\section{Regulatory Submission/Approval}

Once the phase III studies have completed and delivered a positive outcome, compilation of the data to submit to the regulatory agencies starts. This usually takes several months and can be done by one region at a time, e.g. in the United States, or could be done globally, targeting major regions simultaneously. Classically, the major markets include the United States, the European Union and Japan. However, recently more attention is given to the 'emerging markets' such as Latin America, India and China, amongst others. As for the United States, a routine New Drug Application 'NDA' can take up to 15 months for review. However, in cases of particularly high medical need or in areas lacking treatments (e.g. oncology and human immunodeficiency virus), an expedited review can be granted. If the new drug is a biologic, then a biologic license application 'BLA' rather than a 'NDA', is submitted.

In Europe, the sponsor submits a marketing authorisation application (MAA), which could be granted either under the centralised procedure (valid for the entire community market) or through the mutual recognition process.

During the review by the regulatory agencies, questions are referred back to the sponsor. To facilitate the review process, the sponsor will typically establish a rapid response team to coordinate the responses to the authority. Drug label negotiations take place during the review process. Regulatory agencies could request post-approval studies from the drug companies to address any safety concerns that the regulatory agencies may have. At the same time, the drug company will have presented its plans to detect, assess and report adverse events.

Pharmacovigilance is the term used in Europe describing the ongoing evaluation of the safety of the drug in the post-marketing period; it is a requirement that all pharmaceutical companies with a post marketed product must comply. The drug company will also provide periodic safety update reports on the new drug after its approval. Post-marketing or safety surveillance trials are 
sometimes referred to as phase IV clinical trials. Harmful effects discovered during phase IV trials can lead to the withdrawal of the drug from the market as seen in the example of rofecoxib (Vioxx) and cerivastatin (Lipobay, also known as Baycol in the United States).

Orphan Drug Status. Pharmaceutical products developed to treat rare diseases have been referred to as orphan drugs. The FDA Orphan Drug Act specifies the requirements for granting a drug orphan status. The disease that the drug is intended for should affect less than 200,000 people in the United States. This designation grants the company fast-track review process as well as market exclusivity for a period of 7 years. In addition, it will be eligible for direct guidance from the FDA for the design of a clinical plan to further develop the drug. In Europe, some drugs used to treat tropical diseases that are primarily found in developing countries can also be designated as orphan drugs. For the drug companies, the cost of developing such drugs and marketing them will not be covered by the expected sales. Hence, the economic and regulatory incentives to encourage pharmaceutical companies to develop such drugs are needed.

\section{Regulatory Standards}

Preclinical studies are conducted according to good laboratory practice (GLP) guidelines, which regulate how laboratory studies are performed. Clinical trials are conducted according to good clinical practice (GCP) guidelines, which are internationally required quality and safety standards for designing, conducting and reporting clinical trials. GCP-compliant clinical trials are essential to ensure the rights and safety of clinical trial subjects. These standards are subject to inspection by regulatory agencies at any time; regulatory agencies have the right to halt ongoing clinical studies if they have concerns that the studies are not GCP-compliant. Finally drug manufacturing is done according to good manufacturing practice (GMP) guidelines, which dictates the standards for manufacturing and quality control of pharmaceutical products. This is also subject to regulatory inspection.

\section{Lifecycle Management}

The drug company will plan the lifecycle of the drug throughout the patent life and beyond into the future generic marketplace. This may include different drug delivery systems such as prolonged release formulations ver- sus immediate release, combinations with other drugs for improved efficacy, as well as seeking new indications. Once a new indication is confirmed, the drug company can apply for a supplementary new drug application (sNDA). Publication strategies are also another important part of lifecycle management, as additional benefits of the drug that cannot be added to the label, such as patientreported outcome measures, are published in peer-reviewed journals.

\section{Interaction between Pharmaceutical Industry and Healthcare Professionals}

The Pharmaceutical Research and Manufacturers of America (PhRMA) represent research-based pharmaceutical and biotechnology companies. PhRMA have developed guidelines on the basis of interactions between US healthcare professionals and the pharmaceutical industry. The PhRMA code was last updated in January 2009 and regulates amongst other things: informational presentations by pharmaceutical company representatives and accompanying meals, prohibition on entertainment and recreation, pharmaceutical company support for continuing medical education, pharmaceutical company support for third-party educational or professional meetings, the employment of healthcare professionals as consultants, speaker programmes and speaker training meetings, prohibition of non-educational and practicerelated items as well as scholarships and educational funds. In the United Kingdom, the Association of the British Pharmaceutical Industry (ABPI) code was established in 1958 and covers advertising, activities of representatives, supply of samples, provision of hospitality, promotional meetings and the sponsorship of scientific and other meetings, including payment of travelling and accommodation expenses. The ABPI code does not apply to the promotion of over-the-counter medicines to the general public [12].

\section{Conclusion}

Drug development is a long, expensive and highly regulated process. The risks are high, but continued investment in pharmaceuticals is vital if we are to enjoy the benefits of long-term improvements in patient healthcare. 


\title{
References
}

1 Tufts Center for the Study of Drug Development pegs cost of a new prescription medicine at $\$ 802$ million. 2001. http://csdd.tufts. edu/NewsEvents/RecentNews.asp?newsid=6 (accessed January 2, 2009).

2 Drug development: the short story 7. Cost of drug development. Network Science Corporation. http://www.netsci.org/scgi-bin/ Courseware/projector.pl?Course_num $=$ course1\&Filename $=$ slide07.html (accessed January 2, 2009).

3 Drugresearch.com: drug development costs hit $\$ 1.7$ billion. 2003. http://www.drugresearcher.com/Research-management/Drugdevelopment-costs-hit-1.7-billion (accessed January 2, 2009).

4 Kola I, Landis J: Can the pharmaceutical industry reduce attrition rates? Nat Rev Drug Discov 2004;3:711-715.
5 Protección de la propiedad intelectual para productos farmacéuticos: qué es y por qué es esencial para la innovación en salud? Consideraciones a la luz del DR-CAFTA. http:// www.amchamsal.com/uploaded/content/ category/2000065261.pdf (accessed January 2, 2009).

6 Dutta A: Discovery of new medicines; in Griffin JP and O'Grady J (eds): The Textbook of Pharmaceutical Medicine. London, BMJ Books, 2002, p 25

7 International Union of Pure and Applied Chemistry: Glossary of terms used in combinatorial chemistry, U-Z3. Research Triangle Park, International Union of Pure and Applied Chemistry. 1999. http://www.iupac. org/reports/1999/7112maclean/u-z.html (accessed January 2, 2009).
8 Rester U: From virtuality to reality - virtual screening in lead discovery and lead optimization: a medicinal chemistry perspective. Curr Opin Drug Discov Devel 2008;11:559568 .

9 Tweats DJ, Scales MDC: Toxicity testing; in Griffin JP and O'Grady J (eds): The Textbook of Pharmaceutical Medicine. London, BMJ Books, 2002, p 134.

10 Hall JE: The promise of translational physiology. Am J Physiol 2002;283:1235-1236.

11 Biomarkers Definitions Working Group: Biomarkers and surrogate endpoints: preferred definitions and conceptual framework. Clin Pharmacol Ther 2001;69:89-95.

12 What is the code of practice. http://www. pmcpa.org.uk/?q=whatisthecodeofpractice (accessed March 29, 2009).

\section{Editorial Comment}

\author{
M. El Nahas, Sheffield
}

This minireview by Tamimi and Ellis, 2 senior executives at Pfizer UK with considerable experience in drug development and with a genuine interest in nephrology and chronic kidney disease (CKD), is timely. It reminds the reader of the huge and often prohibitive cost of new drug developments. It highlights the fact that thousands of new potentially promising products never make it to the bedside. Giving the cost associated with drug development and the current global financial situation, this minireview sheds considerable light on the direction major pharmaceutical companies may be taking. First, the drug industry is re-evaluating its research priorities moving towards safer clinical areas with projected quicker financial return. Pfizer has moved away from its prior top research priorities and successful drug development areas, namely atherosclerosis and heart failure research. Instead, research and development of drugs to tackle the growing market of Alzheimer's disease are gathering pace. Second- ly, investments in lengthy clinical trials addressing chronic diseases such as CKD may also fall victim to the credit crunch. This editor knows of more than one clinical trial that has been cancelled or stopped as the sponsors' financial situation worsened with the global credit crunch. Finally, drug development may itself be shelved for a more cost-effective approach consisting of acquiring generic drug makers. Over the last few weeks alone, Novartis purchased Ebewe Pharma an Austrian maker of generic cancer drugs for USD 1.3 billion, Pfizer agreed a licencing deal with 2 Indian generic makers and GlaxoSmithKline acquired a stake in South Africa's Aspen. Such an approach may be the way forward for the drug industry to reach a sustainable business model. It may also offer the industry an opportunity to unlock emerging markets that may account for $70 \%$ of new pharmaceutical sales by 2020 . Drugs development and clinical trials have not been spared the ravages of the credit crunch. 